\title{
REVIEW \\ Recent Advances in Studies for the Application of a Protein Produced by Entomopoxviruses (Poxviridae) for Insect-Pest Control
}

\author{
Wataru MITSUHASHI* \\ Division of Insect Sciences, National Institute of Agrobiological Sciences \\ (Tsukuba, Ibaraki 305-8634, Japan)
}

\begin{abstract}
The use of insect-virus insecticides has been very limited and the development of new insecticides has not been pursued actively. This is mainly because the cost of pest control using the insecticides is very high considering its effect compared with the cost versus effect of using synthetic insecticides. One way of diffusing insect-virus insecticides is to strengthen the ability of the viruses in the formulation to kill pests. Strengthening the virus activity will lead to a drastic reduction in the amount applied per unit area, cost of control will be lowered, and the use of such insecticides will spread. Several groups have studied the synergistic effect of an entomopoxvirus protein, fusolin, on the infectivity of insect viruses to strengthen their ability to kill pests. Fusolin is the main component of entomopoxvirus inclusion bodies called spindles, and the spindles of several entomopoxviruses have been found to strongly enhance many nucleopolyhedroviruses. The enhancement occurs through the disruption of the peritrophic membrane, a barrier against virus infection, by the spindles. Two of these fusolin genes were cloned, and two and one of these genes were expressed in foreign gene expression systems and a crop species, respectively. As a result, the gene products enhanced viral infection. Finally, the functional region for the enhancement in this gene has been shown and the detailed mode of action of fusolin was estimated.
\end{abstract}

Discipline: Insect pest

Additional key words: Anomala cuprea, fusolin, nucleopolyhedrovirus, peritrophic membrane, spindle

\section{Introduction}

It has been hoped that insect-virus insecticides have a wider use in agriculture and forestry, because they are safe for vertebrates, including humans and livestock, most beneficial insects, plants, and the environment. Baculoviruses, which include all viruses of the family Baculoviridae, are considered to be especially safe as components of insectviral insecticides because they infect only arthropods. Most insect viruses that have been used as components of insect-virus insecticides are members of the baculoviruses composed of nucleopolyhedroviruses (NPVs) and granuloviruses (GVs).

The share of insect-virus insecticides sold (hereafter referred to as viral insecticides) in the total amount of the insecticides market has been very small, especially in developed countries. In Japan only four kinds of viral insecticide have been registered and currently three are available; those with trade names Hamaki-Tenteki and Kayaku Hamaki-Tenteki are composed of Adoxophyes orana GV and Homona magnanima GV for A. honmai and H. magnanima, and Hasumon Tenteki is composed of Spodoptera litura NPV for the common cutworm, S. litura. The main reasons for the limited use are as follows. (1) The commercial production costs of viral insecticides are much higher than those of synthetic insecticides because their production is much more labor-intensive. (2) The spectrum of pests killed by each virus species is narrow, so it may be necessary to use a different viral insecticide for each insect or group of insects. (3) The period from infection with viruses to death is relatively long, and during that time, the pests continue to consume the crops. However, a drastic reduction in the very high costs for pest control because of the above reasons may lead to the expansion of viral insecticides, and strengthening the ability of the

*Corresponding author: e-mail mitsuhas@affrc.go.jp

Received 26 November 2008; accepted 13 January 2009. 
viruses contained in the formulations to kill pests has been attempted. There are two major methods for strengthening the ability of the viruses. One is the use of recombinant baculoviruses constructed by introducing foreign genes that play roles in increasing the speed of killing and/or expanding the host range into the virus genome. However, no recombinant baculoviruses have been registered as viral insecticides owing to a lack of consensus on the use of such genetically modified organisms in fields. The second method is the use of synergistic agents that can enhance the peroral infectivity of viruses by oral administration, thereby reducing the amount of viruses needed per unit area. This could lower the costs of pest control with viruses and promote the use of viral insecticides. In fact, researchers have found more than 10 synergists from synthetic compounds and proteins of insect viruses. Moreover, natural synergists, in general, are likely to be less harmful to vertebrates and the environment than synthetic compounds.

This article reviews the studies on one synergist of viral insecticides, fusolin, which is a major constituent protein of paracrystaline structures (inclusion bodies) called spindles (Fig. 1A), produced by entomopoxviruses (EPVs). This study field arose in Japan and a group including me has extensively contributed to the advances in the field.

\section{EPVs}

EPVs include all viruses of the subfamily Entomopoxvirinae in the family Poxviridae, and EPVs infect insects. The Entomopoxvirinae are subdivided into the following three genera: Alphaentomopoxvirus whose viruses infect Coleoptera, Betaentomopoxvirus whose viruses infect Lepidoptera and Orthoptera, and Gammaentomopoxvirus of Diptera. EPV virions are ovoid or brick-shaped (220-265 $\mathrm{nm} \times 270-470 \mathrm{~nm})$ and contain a large, linear, double-stranded DNA of 225-380 kbp. EPVs replicate mainly in the fat bodies of hosts and form two types of inclusion bodies, spheroids and spindles, in the cytoplasm of host cells (Fig. 1). Almost all EPVs form spheroids ${ }^{6}$. However, Gammaentomopoxvirus species and some Betaentomopoxvirus species do not form spindles. Very small spindles of some lepidopteran EPVs are occluded in spheroids ${ }^{1,2}$, although those of coleopteran EPVs are not occluded ${ }^{6}$. The major constitutive protein of the spheroids is spheroidin. Spheroids contain virions ${ }^{19}$ (Fig. 1C), whereas the spindles do not. In fields, spheroids may play a role in the protection of virions against sunlight (ultraviolet radiation) and high temperature ${ }^{1}$, and the function of spindles may be to enhance EPV infectivity ${ }^{11,14,21,22}$.

\section{Enhancement of virus infectivity by spindles}

The first study concerning this field, i.e. the enhancement of virus infection by the protein from EPVs, was reported from Japan as mentioned above by Xu \& Hukuhara $(1992)^{25}$. They reported that the infectivity of Pseudaletia unipuncta NPV (PuNPV) in P. separata larvae (Lepidoptera) was strongly enhanced by peroral administration of the spheroids from Pseudaletia separata EPV (PsEPV) or a solution of the spheroids. The viruses were administered in a status occluded by polyhedra.

Mitsuhashi et al. (1998) ${ }^{13}$ found that spindles of an EPV isolated from the scarabaeid beetle Anomala cuprea (AcEPV), but not the spheroids, strongly enhanced the infectivity of the polyhedra of Bombyx mori NPV (BmNPV)



A

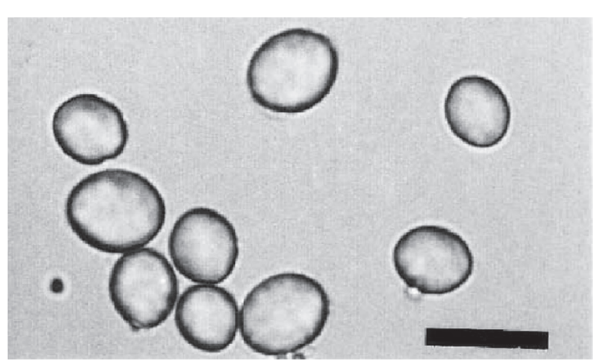

B

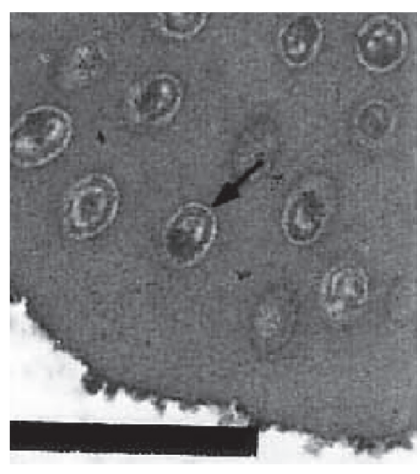

C

Fig. 1. Anomala cuprea entomopoxvirus inclusion bodies

A: Spindles. B: Spheroids. C: Sectional view of the spheroid. The arrow indicates a virion. The virion shows the characteristics of genus Alphaentomopoxvirus, in which the core is uniconcave. Bars in A and B indicate $10 \mu \mathrm{m}$ and that in $\mathrm{C}, 1 \mu \mathrm{m}$. 
in B. mori larvae (Fig. 2, Table 1). Wijonarko \& Hukuhara $(1998)^{24}$ also showed that not only the spheroids of PsEPV but also its spindles and virions enhanced the infectivity of PuNPV polyhedra in P. separata larvae. Furthermore several reports have shown that AcEPV or Helicoverpa armigera EPV spindles enhanced the infectivity of the polyhedra of other NPV species ${ }^{3,20}$.

Based on bioassays using AcEPV spheroids and its

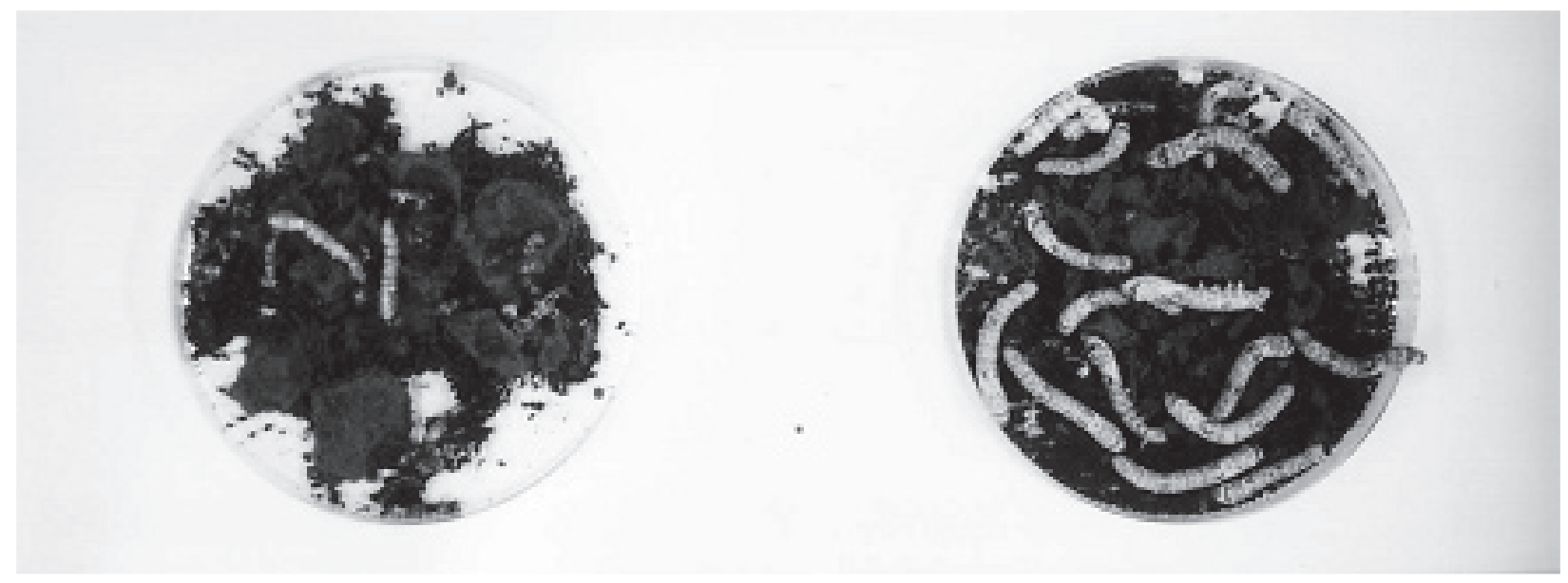

Fig. 2. Bombyx mori larvae at 5 days after the termination of the administration of Bombyx mori nucleopolyhedrovirus (BmNPV) polyhedra with or without Anomala cuprea entomopoxvirus (AcEPV) spindles

Twenty third-instar larvae were group-administered with BmNPV polyhedra with or without AcEPV spindles in an artificial diet, and the diet was consumed completely. The average numbers of BmNPV polyhedra and AcEPV spindles consumed by each larva were as follows. Left dish: polyhedra, 10; spindles; $2.5 \times 10^{6}$. Right: polyhedra alone, $1 \times 10^{5}$. Twenty larvae in each group were then transferred to a dish containing fresh artificial diet. All of the larvae fed with spindles died, and most darkened and were difficult to recognize in the image. Conversely, most of the larvae fed polyhedra alone remained alive and were clearly recognizable.

Table 1. Degree of the enhancement of the infectivity of Bombyx mori nucleopolyhedrovirus $(\mathrm{BmNPV})$ in B.mori $(\mathrm{C} 146 \times \mathrm{N} 137)$ larvae by Anomala cuprea entomopoxvirus spindles and the stability of their activity against heating

\begin{tabular}{|c|c|c|c|c|c|c|c|c|c|}
\hline \multicolumn{2}{|c|}{ Inoculum } & \multicolumn{7}{|c|}{ No. of dead larvae $(\mathrm{N}=20)^{\mathrm{a}}$} & \multirow[t]{3}{*}{$\log \operatorname{LD}_{50}{ }^{b}$} \\
\hline & \multirow{2}{*}{$\begin{array}{l}{ }^{\circ} \mathrm{C} \text { at which } \\
\text { spindles were } \\
\text { heated } \\
\text { for } 30 \mathrm{~min}\end{array}$} & \multicolumn{7}{|c|}{ No. of BmNPV polyhedra inoculated per larva } & \\
\hline & & 0 & $1 \times 10^{1}$ & $1 \times 10^{2}$ & $1 \times 10^{3}$ & $1 \times 10^{4}$ & $1 \times 10^{5}$ & $1 \times 10^{6}$ & \\
\hline $\begin{array}{l}\text { BmNPV } \\
\text { polyhedra alone }\end{array}$ & & 0 & $\mathrm{nt}$ & $\mathrm{nt}$ & 0 & 0 & 4 & 12 & $\geq 5.70 \pm 0.29$ \\
\hline \multirow{4}{*}{$\begin{array}{l}\text { Spindles } \\
\text { combined } \\
\text { with BmNPV } \\
\text { polyhedra }\end{array}$} & 95 & 0 & 20 & 20 & 20 & 20 & $\mathrm{nt}^{\mathrm{c}}$ & $\mathrm{nt}$ & $\leq 0.50 \pm 0.00$ \\
\hline & 85 & $0^{\mathrm{d}}$ & 19 & 20 & 20 & 20 & $\mathrm{nt}$ & $\mathrm{nt}$ & $\leq 0.55 \pm 0.10$ \\
\hline & 75 & 0 & 20 & 20 & 20 & 20 & nt & $\mathrm{nt}$ & $\leq 0.50 \pm 0.00$ \\
\hline & No heating & 0 & 19 & 20 & 20 & 20 & $\mathrm{nt}$ & $\mathrm{nt}$ & $\leq 0.55 \pm 0.10$ \\
\hline
\end{tabular}

a: $\mathrm{N}$ means the number of larvae tested in each treatment of larvae.

b: $\log \mathrm{LD}_{50}$ was expressed by the $95 \%$ confidence limit of the Spearman-Kärber method (Finney, 1964) ${ }^{4}$.

c: Not tested.

d: No. of tested larvae was 19 in this treatment of larvae (inoculum; spindles heated at $85{ }^{\circ} \mathrm{C}$ without polyhedra).

Each spindle sample was heated in a water or block bath (block bath for $95{ }^{\circ} \mathrm{C}$ only).

The number of spindles inoculated perorally per third-instar larva of the first day, i.e., day 0 , was $1 \times 10^{6}$.

Different from the bioassays shown in Fig.2, larvae were individually inoculated and individually reared after the inoculation. 
spindles, EPV spindles have been demonstrated to enhance its infectivity, and facilitating EPV infection is thus thought to be the natural biological function of the spindles $^{11,14,21,22}$.

NPVs have another phenotype of virions, a non-occluded virus (budded virus) (NOV). NOVs are not occluded by polyhedra and are rarely infectious through the insect midgut when administered perorally. However, Furuta et al. (2001) $)^{5}$ showed that NOVs of BmNPV (both of wild type NPV and polyhedrin-negative recombinant NPV) become highly infectious perorally to $B$. mori larvae when administered with AcEPV spindles. This finding suggests that spindles could be a strong tool for mass-production biofactories using the recombinant viruses. This is because inoculating these recombinant viruses perorally reduces greatly the amount of labor substantially compared with that in the conventional inoculation method that is to inject the viruses into host hemocoel one by one.

The enhancement of NPV infectivity by AcEPV spindles is highly stable against various abiotic factors such as high temperature, ultraviolet radiation, formaldehyde, ethyl alcohol, and the bactericide benzalkonium chloride ${ }^{17}$. For example, AcEPV spindles retain high activity even after being heated at $95^{\circ} \mathrm{C}$ for $30 \mathrm{~min}$ (Table 1), while synergistic activity of Pseudaletia unipuncta $\mathrm{GV}$ granules for NPV infectivity was lost by heating them at $85^{\circ} \mathrm{C}$ for $10 \mathrm{~min}^{23}$. This nature of the spindles is a desirable trait as a synergist of viral insecticides.

\section{Mode of action of infectivity enhancement}

The conformation of normal peritrophic membrane (PM), which is an acellular, tube- or bag-like membrane lining the midgut lumen and extending from the anterior midgut to the hindgut, in B. mori appeared to physically prevent the passage of BmNPV to a considerable degree ${ }^{16}$, and the PM of B. mori larvae was disintegrated by administering AcEPV spindles ${ }^{15}$. Thus the disintegration of the PM is thought to be one mode of action by which EPV spindles enhance NPV infectivity. Furthermore, the PM of A. cuprea larvae was disrupted by feeding of AcEPV spindles, thereby facilitating infection of the host's midgut by the $\mathrm{EPV}^{14}$. This strongly suggests that the mode of action of the enhancement of viral infection is similar in NPV and EPV infections. Hukuhara \& Wijonarko (2001) ${ }^{10}$ and Hukuhara et al. (2001) $)^{9}$ showed that an enhancing factor called EF (namely PsEPV fusolin) enhanced the fusion between PuNPV virions and cultured insect cells derived from hemocytes, and speculated that the infection of $P$. separata larvae by the NPV was enhanced due to the enhanced fusion of the virions to the midgut cells mediated by the EF. Thus, this enhanced fusion may be another mode of action of the spindles (fusolin) by which NPV infection is enhanced; further studies are required to investigate whether this phenomenon occurs in vivo.

\section{Analyses of fusolin}

The EF in PsEPV spheroids that enhances the infectivity of PuNPV was reported to be a $38-\mathrm{kDa}$ glycoprotein $^{26}$. The gene that codes for the EF was cloned from the PsEPV genome and it turned out to be the PsEPV fusolin gene $^{7}$. Furthermore, AcEPV fusolin was cloned and its nucleotide sequence was determined ${ }^{18}$. In fusolins from different various EPVs, their determined nucleotide sequences and deduced amino acid sequences were reported, but there have not been reports concerning the presence or absence of the ability of the fusolins or spindles to enhance virus infectivity ${ }^{12}$.

Fusolins vary from about 340 to 390 amino acid residues, and the N-terminus (about 15-20 aa residues) is a signal peptide that is cleaved from the protein to produce the mature form ${ }^{12}$. This cleavage suggests that fusolin moves to the endoplasmic reticulum and crystallizes there.

My group has identified the region of AcEPV fusolin, which is involved in the viral enhancing activity by assaying the activity of various AcEPV fusolin mutants using a bioassay system including BmNPV and $B$. mori larvae ${ }^{22}$. Namely, the N-terminal region (ca. 250 amino acid residues from the $\mathrm{N}$-terminus of a translated form) containing a signal peptide is essential for the activity and N-glycosylation is important; the rest of the fusolin is non-essential. It was found that the $\mathrm{N}$-terminal region binds to chitin in vitro and that glycosylation is important for chitin binding $^{22}$. This essential region contains some conserved regions among fusolins and GP37s of baculoviruses, and the regions constitute a chitin-binding domain. This domain has been specifically classified into chitin-binding domain 3 (InterPro database). Therefore this domain should bind to chitin, and this binding should be associated with PM disruption, since one main component of the PM is chitin. In addition, the non-essential region is easily eliminated by serine protease(s) in insect digestive juice, whereas the essential region is more stable, and the glycosylation is required for the stability of the $\mathrm{N}$-terminal region ${ }^{22}$. A diagram of the structure of AcEPV fusolin showing the functional region is illustrated in Fig. 3.

\section{Developing efficient production-methods of fusolin for its practical use}

It is not necessarily a good method to use host insects for the mass production of fusolin. Such production is usually very labor-intensive, because the approach requires 


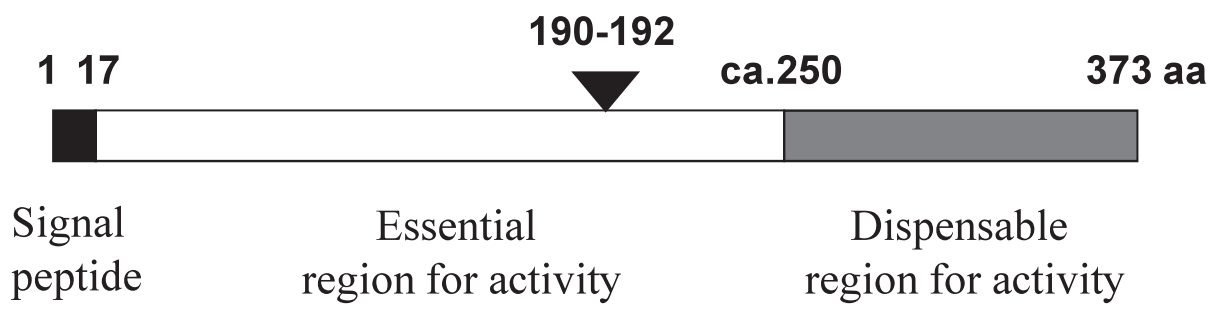

$\nabla:$ N-glycosylation site

Fig. 3. Structure of Anomala cuprea entomopoxvirus fusolin

The carbohydrate chain is important for the enhancing-activity. Essential region for activity; this region can bind chitin, suggesting the mechanism of the disruption of the peritrophic membrane (see text). Dispensable region for activity; this region is easily digested in an insect midgut juice. aa: amino acid.

mass-rearing of insects followed by protein purification, and this increases production costs. Thus, an alternative method, i.e. the use of other organisms that require less labor and time to rear or culture, or in vitro production, is needed for the practical production of the protein. The use of crop plants in fields, into which the gene was introduced and in which the expression of the protein occurs, is another potential method, since it merits the omission of preparing the protein as an additive of viral insecticides.

Hukuhara et al. (1999) ${ }^{8}$ introduced a fusolin gene isolated from PsEPV into rice cultivars and observed approximately a 360-fold enhancement of PuNPV infectivity in P. separata larvae that were fed on the transgenic plants containing the fusolin. Furthermore, Hukuhara et al. $(2001)^{9}$ expressed a PsEPV fusolin gene that had been introduced into E. coli, and it was found that the protein produced by this gene enhanced PuNPV infection in $P$. separata larvae by nearly 270 -fold. My group has expressed the AcEPV fusolin gene in a baculovirus foreign gene expression system, and found that the resulting protein enhanced BmNPV infection of B. mori third-instar larvae by about 320 -fold, but the degree of the enhancement appeared higher in the case of second instar larvae ${ }^{22}$. Furthermore, the enhancement of the infectivity of AcEPV by AcEPV fusolin produced by a baculovirus expression system was observed in A. cuprea larvae ${ }^{22}$. The degrees of infectivity enhancement by the alternative methods were markedly lower in many cases than those achieved using spindles. From the practical perspective, it is important to elucidate the cause of this phenomenon.

\section{Conclusion}

There are some pests whose infection by NPVs is not affected by the disruption of the PMs by tinopal. It is a possibility that these species possess larger pores than
NPVs in the PMs, and thus the PMs do not act as a barrier against NPVs ${ }^{16}$. In contrast, the PMs of most pests may play a role in preventing the passages of NPVs through the PMs to a considerable degree, and thus fusolin may be available as a synergistic agent for many kinds of viral insecticides.

As described above, a reduction in the use of synthetic pesticides in the field has been anticipated to have benefits for human health and the prevention of environmental pollution, and viral insecticides are promising tools as alternatives to synthetic pesticides. It is not clear if popular use of recombinant insect viruses that show a strong ability to kill pests will be realized in the near future. Therefore, it is important to continue intensive research to produce practical synergistic viral proteins, such as fusolin, and the most important theme may be to develop efficient production methods of fusolin for its practical use.

\section{Acknowledgments}

I thank my colleagues, especially Mamoru Sato, Yoji Furuta, Hiromu Kawakita, Hitoshi Saito, Kazuhisa Miyamoto and Ritsuko Murakami, for their collaboration with me in the studies described here.

\section{References}

1. Arif, B. M. (1995) Recent advances in the molecular biology of entomopoxviruses. J. Gen. Virol., 76, 1-13.

2. Bird, F. T. (1974) The development of spindle inclusions of Choristoneura fumiferana (Lepidoptera: Tortricidae) infected with entomopox virus. J. Invertebr. Pathol., 23, 325-332.

3. Chakraborty, M., Narayanan, K. \& Sivaprakash, M. K. (2004) In vivo enhancement of nucleopolyhedrovirus of oriental armyworm, Mythimna separata using spindles from Helicoverpa armigera entomopoxvirus. Indian $J$. 
Exp. Biol., 42, 121-123.

4. Finney, D. J. (1964) Statistical methods in biological assays, 2nd ed. Charles Griffin, London, UK.

5. Furuta, Y. et al. (2001) Peroral infectivity of non-occluded viruses of Bombyx mori nucleopolyhedrovirus and polyhedrin-negative recombinant baculoviruses to silkworm larvae is drastically enhanced when administered with Anomala cuprea entomopoxvirus spindles. J. Gen. Virol., 82, 307-312.

6. Gauthier, L. et al. (1995) The Melolontha melolontha entomopoxvirus (MmEPV) fusolin is related to the fusolins of lepidopteran EPVs and to the $37 \mathrm{~K}$ baculovirus glycoprotein. Virology, 208, 427-436.

7. Hayakawa, T., Xu, J. \& Hukuhara, T. (1996) Cloning and sequencing of the gene for an enhancing factor from $\mathrm{Pseu}$ daletia separata entomopoxvirus. Gene, 177, 269-270.

8. Hukuhara, T., Hayakawa, T. \& Wijonarko, A. (1999) Increased baculovirus susceptibility of armyworm larvae feeding on transgenic rice plants expressing an entomopoxvirus gene. Nat. Biotechnol., 17, 1122-1124.

9. Hukuhara, T., Hayakawa, T. \& Wijonarko, A. (2001) A bacterially produced virus enhancing factor from an entomopoxvirus enhances nucleopolyhedrovirus infection in armyworm larvae. J. Invertebr. Pathol., 78, 25-30.

10. Hukuhara, T. \& Wijonarko, A. (2001) Enhanced fusion of a nucleopolyhedrovirus with cultured cells by a virus enhancing factor from an entomopoxvirus. J. Invertebr. Pathol., 77, 62-67.

11. Mitsuhashi, W. (2002) Further evidence that spindles of an entomopoxvirus enhance its infectivity in a host insect. $J$. Invertebr. Pathol., 79, 59-61.

12. Mitsuhashi, W. (2006) Enhancement of the infectivity of insect viruses: a protein produced by entomopoxviruses is a potential synergist of viral pesticides. In Recent research developments in entomology, vol. 5, ed. Pandalai, S. G., Research Signpost, Kerala, India, 147-159.

13. Mitsuhashi, W., Furuta, Y. \& Sato, M. (1998) The spindles of an entomopoxvirus of Coleoptera (Anomala cuprea) strongly enhance the infectivity of a nucleopolyhedrovirus in Lepidoptera (Bombyx mori). J. Invertebr. Pathol., 71, 186-188.

14. Mitsuhashi, W. et al. (2007) Spindles of an entomopoxvirus facilitate its infection of the host insect by disrupting the peritrophic membrane. J. Virol., 81, 4235-4243.

15. Mitsuhashi, W. \& Miyamoto, K. (2003) Disintegration of the peritrophic membrane of silkworm larvae due to spindles of an entomopoxvirus. J. Invertebr. Pathol., 82, 34-40.

16. Mitsuhashi, W. \& Murakami, R. (2007) Scanning electron microscopic observations of the peritrophic membrane in silkworm (Bombyx mori) larvae. Trans. Lepid. Soc. Japan, 58, 172-176.

17. Mitsuhashi, W. et al. (2008) Stability of the viral-enhancing ability of entomopoxvirus spindles exposed to various abiotic factors. Appl. Entomol. Zool., 43, 483-489.

18. Mitsuhashi, W., Saito, H. \& Sato, M. (1997) Complete nucleotide sequence of the fusolin gene of an entomopoxvirus in the cupreous chafer, Anomala cuprea Hope (Coleoptera: Scarabaeidae). Insect Biochem. Mol. Biol., 27, 869-876.

19. Mitsuhashi, W. et al. (1998) Complete nucleotide sequence of spheroidin gene of Anomala cuprea entomopoxvirus. Virus Res., 55, 61-69.

20. Mitsuhashi, W. \& Sato, M. (2000) Enhanced infection of a nucleopolyhedrovirus in a lepidopteran pest (Spilosoma imparilis) by spindles of a coleopteran entomopoxvirus (EPV) (Anomala cuprea EPV). J. For. Res., 5, 285-287.

21. Mitsuhashi, W., Sato, M. \& Hirai, Y. (2000) Involvement of spindles of an entomopoxvirus (EPV) in infectivity of the EPVs to their host insect. Arch. Virol., 145, 1465-1471.

22. Takemoto, Y. et al. (2008) The N-terminal region of an entomopoxvirus fusolin is essential for the enhancement of peroral infection, whereas the C-terminal region is eliminated in digestive juice. J. Virol., 82, 12406-12415.

23. Tanada, Y. (1959) Synergism between two viruses of the armyworm, Pseudaletia unipuncta (Haworth) (Lepidoptera, Noctuidae). J. Invertebr. Pathol., 1, 215-231.

24. Wijonarko, A. \& Hukuhara, T. (1998) Detection of a virus enhancing factor in the spheroid, spindle, and virion of an entomopoxvirus. J. Invertebr. Pathol., 72, 82-86.

25. Xu, J. \& Hukuhara, T. (1992) Enhanced infection of a nuclear polyhedrosis virus in larvae of the armyworm, Pseudaletia separata, by a factor in the spheroids of an entomopoxvirus. J. Invertebr. Pathol., 60, 259-264.

26. Xu, J. \& Hukuhara, T. (1994) Biochemical properties of an enhancing factor of an entomopoxvirus. J. Invertebr. Pathol., 63, 14-18. 